\title{
ASYMPTOTIC SOLUTION OF THE HIGH ORDER PARTIAL DIFFERENTIAL EQUATION
}

\author{
${ }^{1}$ Hoang Van Da, ${ }^{1}$ Tran Dinh Son, ${ }^{2}$ Nguyen Duc Tinh \\ ${ }^{1} \mathrm{Ha}$ Noi University of Mining and Geology \\ ${ }^{2}$ Quang Ninh University of Industry
}

\begin{abstract}
In the present paper, the authors have constructed an asymptotic solution of the high order equation with partial derivatives by means of the asymptotic method for the high order systems. The improved first approximation of the solution of the given boundary value problem is determined.
\end{abstract}

\section{INTRODUCTION}

The problem of the oscillation of the creepy elastic beam with linear boundary conditions in the autonomous case has been studied [3]. In this work, the authors inverstigate the oscillation of the creepy elastic beam, in the non-autonomous case, described by the third order equation as follows:

$$
\frac{\partial^{3} y}{\partial t^{3}}+\xi \frac{\partial^{2} y}{\partial t^{2}}+\omega^{2} \frac{\partial^{5} y}{\partial t \partial x^{4}}+\xi \omega^{2} \frac{\partial^{4} y}{\partial x^{4}}=\varepsilon F(x, y, \dot{y}, \theta, \ldots),
$$

where $\xi, \omega, \Omega$ are real constants, $\varepsilon$ is a small parameter, $\theta=\theta(t), y=y(x, t)$. The relevant homogeneous boundary conditons are

$$
\left.y\right|_{x=0}=0,\left.\quad \frac{\partial^{2} y}{\partial x^{2}}\right|_{x=0}=0,\left.\quad y\right|_{x=0}=0,\left.\quad \frac{\partial^{2} y}{\partial x^{2}}\right|_{x=0}=0 .
$$

It is supposed that the resonance relation takes the from.

$$
\begin{gathered}
\Omega_{1}=\frac{p}{q} \gamma+\varepsilon \Delta, \\
\frac{d \theta}{d t}=\gamma,
\end{gathered}
$$

$p, q$ are integers.

\section{CONTRUCTION OF THE NON-AUTONOMOUS SYSTEM}

With the boundary conditions (2) we get the fundamental functions and the eigenvalues in the form

$$
Z_{k}(x)=\sin \frac{k \pi x}{\ell}, \beta_{k}=\frac{k^{2} \pi^{2}}{l^{2}} \quad(k=1,2, \ldots) .
$$


In this case, the partial solution of the equation (1) is found in form of the following series [6]

$$
\begin{gathered}
y(x, t)=a \cos \varphi Z_{1}+\varepsilon U_{1}(x, a, \varphi, \theta)+\varepsilon^{2} U_{2}(x, a, \varphi, \theta)+\ldots \\
\varphi=\left(\frac{p}{q} \theta+\psi\right)
\end{gathered}
$$

where $a, \psi$ are the functions satisfy the following differential equations

$$
\begin{gathered}
\frac{d a}{d t}=\varepsilon A_{1}(a, \psi)+\varepsilon^{2} A_{2}(a, \psi)+\ldots \\
\frac{d \psi}{d t}=\left(\Omega_{1}-\frac{p}{q \gamma}\right)+\varepsilon B_{1}(a, \psi)+\varepsilon^{2} B_{2}(a, \psi)+\ldots
\end{gathered}
$$

Now differentiating the function $y(x, t)$ in the form (6) with respect to argument $t$, after calculation we get

$$
\begin{aligned}
& \frac{\partial y}{\partial t}=\varepsilon A_{1} \cos \varphi Z_{1}-a \Omega_{1} \sin \varphi Z_{1}-\varepsilon \beta_{1} a \sin \varphi Z_{1}+\varepsilon\left(\Omega_{1} \frac{\partial U_{1}}{\partial \varphi}+\gamma \frac{\partial U_{1}}{\partial \theta}\right) \\
& \frac{\partial^{2} y}{\partial t^{2}}=\varepsilon \frac{\partial A_{1}}{\partial \psi} \frac{d \psi}{d t} \cos \varphi Z_{1}-\varepsilon A_{1} \sin \varphi \frac{d \varphi}{d t} Z_{1}-\frac{d a}{d t} \Omega_{1} \sin \varphi Z_{1}-a \Omega_{1} \frac{d \varphi}{d t} \cos \varphi Z_{1} \\
& -\varepsilon \frac{\partial B_{1}}{\partial \psi} \frac{d \psi}{d t} a \sin \varphi Z_{1}-\varepsilon B_{1} a \cos \varphi \frac{d \varphi}{d t} Z_{1}+\varepsilon\left(\Omega_{1}^{2} \frac{\partial^{2} U_{1}}{\partial \varphi^{2}}+2 \Omega_{1} \gamma \frac{\partial^{2} U_{1}}{\partial \varphi \partial \theta}+\gamma^{2} \frac{\partial^{2} U_{1}}{\partial \theta^{2}}\right) \\
& \frac{\partial^{2} y}{d t^{2}}=\varepsilon \frac{\partial A_{1}}{\partial \psi}\left(\Omega_{1}-\frac{p}{q} \gamma\right) \cos \varphi Z_{1}-\varepsilon A_{1} \Omega_{1} \sin \varphi Z_{1}-\varepsilon A_{1} \Omega_{1} \sin \varphi Z_{1}-\varepsilon \Omega_{1}^{2} \cos \varphi Z_{1} \\
& -\varepsilon B_{1} a \Omega_{1} \cos \varphi Z_{1}-\varepsilon \frac{\partial B_{1}}{\partial \psi}\left(\Omega_{1}-\frac{p}{q} \gamma\right) a \sin \varphi Z_{1}-\varepsilon B_{1} a \Omega_{1} \cos \varphi Z_{1} \\
& +\varepsilon\left(\Omega_{1}^{2} \frac{\partial^{2} U_{1}}{\partial \varphi^{2}}+2 \Omega_{1} \gamma \frac{\partial^{2} U_{1}}{\partial \varphi \partial \theta}+\gamma^{2} \frac{\partial^{2} U_{1}}{\partial \theta^{2}}\right) \\
& \frac{\partial^{2} y}{\partial t^{2}}=\varepsilon \frac{\partial A_{1}}{\partial \psi}\left(\Omega_{1}-\frac{p}{q} \gamma\right) \cos \varphi Z_{1}-2 \varepsilon A_{1} \Omega_{1} \sin \varphi Z_{1}-2 \varepsilon B_{1} a \Omega_{1} \cos \varphi Z_{1} \\
& -\varepsilon \frac{\partial B_{1}}{\partial \psi}\left(\Omega_{1}-\frac{p}{q} \gamma\right) a \sin \varphi Z_{1}-a \Omega_{1}^{2} \cos \varphi Z_{1}+\varepsilon\left(\Omega_{1}^{2} \frac{\partial^{2} U_{1}}{\partial \varphi^{2}}+2 \Omega_{1} \gamma \frac{\partial^{2} U_{1}}{\partial \varphi \partial \theta}+\gamma^{2} \frac{\partial^{2} U_{1}}{\partial \theta^{2}}\right) . \\
& \frac{\partial^{3} y}{\partial t^{3}}=\varepsilon \frac{\partial^{2} A_{1}}{\partial \psi^{2}}\left(\Omega_{1}-\frac{p}{q} \gamma\right)^{2} \cos \varphi Z_{1}-\varepsilon \frac{\partial A_{1}}{\partial \psi}\left(\Omega_{1}-\frac{p}{q} \gamma\right) \sin \varphi \Omega_{1} Z_{1} \\
& -2 \varepsilon \frac{\partial A_{1}}{\partial \psi^{2}}\left(\Omega_{1}-\frac{p}{q} \gamma\right) \Omega_{1} \sin \varphi Z_{1}-2 \varepsilon A_{1} \Omega_{1}^{2} \cos \varphi Z_{1}-2 \varepsilon \frac{\partial B_{1}}{\partial \psi}\left(\Omega_{1}-\frac{p}{q} \gamma\right) a \Omega_{1} \cos \varphi Z_{1} \\
& +2 \varepsilon B_{1} a \Omega_{1}^{2} \sin \varphi Z_{1}-\varepsilon \frac{\partial^{2} B_{1}}{\partial \psi^{2}}\left(\Omega_{1}-\frac{p}{q} \gamma\right)^{2} a \sin \varphi Z_{1}-\varepsilon \frac{\partial B_{1}}{\partial \psi}\left(\Omega_{1}-\frac{p}{q} \gamma\right) a \Omega_{1} \cos Z_{1} \\
& -\varepsilon A_{1} \Omega_{1}^{2} \cos \varphi Z_{1}+a \Omega_{1}^{3} \sin \varphi Z_{1}^{\dagger}+\varepsilon B_{1} a \Omega_{1}^{2} \sin \varphi Z_{1}+\varepsilon\left(\Omega_{1}^{3} \frac{\partial^{3} U_{1}}{\partial \varphi^{3}}+3 \Omega_{1} \gamma \frac{\partial^{3} U_{1}}{\partial \varphi^{2} \partial \theta}\right.
\end{aligned}
$$


$\left.+3 \Omega_{1} \gamma \frac{\partial^{3} U_{1}}{\partial \varphi \partial \theta^{2}}+\gamma^{3} \frac{\partial^{3} U_{1}}{\partial \theta^{3}}\right)$

$\frac{\partial^{3} y}{\partial t^{3}}=\varepsilon \frac{\partial^{2} A_{1}}{\partial \psi^{2}}\left(\Omega_{1}-\frac{p}{q} \gamma\right)^{2} \cos \varphi Z_{1}-3 \varepsilon \frac{\partial A_{1}}{\partial \psi}\left(\Omega_{1}-\frac{p}{q} \gamma\right) \Omega_{1} \sin \varphi Z_{1}-3 \varepsilon A_{1} \Omega_{1}^{2} \cos \varphi Z_{1}$

$-\varepsilon \frac{\partial^{2} B_{1}}{\partial \psi^{2}}\left(\Omega_{1}-\frac{p}{q} \gamma\right)^{2} a \sin \varphi Z_{1}-3 \varepsilon \frac{\partial B_{1}}{\partial \psi}\left(\Omega_{1}-\frac{p}{q} \gamma\right) a \Omega_{1} \cos \varphi Z_{1}+3 \varepsilon B_{1} a \Omega_{1}^{2} \sin \varphi Z_{1}$

$+a \Omega_{1}^{3} \sin \varphi Z_{1}+\varepsilon\left(\Omega_{1}^{3} \frac{\partial^{3} U_{1}}{\partial \varphi^{3}}+3 \Omega_{1}^{2} \gamma \frac{\partial^{3} U_{1}}{\partial \varphi^{2} \partial \theta}+3 \Omega_{1} \gamma^{2} \frac{\partial^{3} U_{1}}{\partial \varphi \partial \theta^{2}}+\gamma^{3} \frac{\partial^{3} U_{1}}{\partial \theta^{3}}\right)$.

Now we calculate some of quantities in the equation (1).

$$
\begin{aligned}
\xi \frac{\partial^{2} y}{\partial t^{2}}= & \varepsilon \xi \frac{\partial A_{1}}{\partial \psi}\left(\Omega_{1}-\frac{p}{q} \gamma\right) \cos \varphi Z_{1}-2 \varepsilon \xi A_{1} \Omega_{1} \sin \varphi Z_{1}-2 \varepsilon \xi B_{1} \Omega_{1} a \cos \varphi Z_{1} \\
- & \varepsilon \xi \frac{\partial B_{1}}{\partial \psi}\left(\Omega_{1}-\frac{p}{q} \gamma\right) a \sin \varphi Z_{1}-\xi a B_{1} \Omega_{1}^{2} \cos \varphi Z_{1}+\varepsilon \xi L_{2}\left[U_{1}\right] \\
\omega^{2} \frac{\partial^{5} y}{\partial t \partial x^{4}}= & \varepsilon A_{1} \omega^{2} \frac{\pi^{4}}{\ell^{4}}\left(\Omega_{1}-\frac{p}{q} \gamma\right) \cos \varphi Z_{1}-a \Omega_{1} \omega^{2} \frac{\pi^{4}}{\ell^{4}} \sin \varphi Z_{1}-\varepsilon B_{1} a \omega^{2} \frac{\pi^{4}}{\ell^{4}} \sin \varphi Z_{1} \\
& +\varepsilon\left(\omega^{2} \Omega_{1} \frac{\partial^{5} U_{1}}{\partial \varphi \partial x^{4}}+\omega^{2} \gamma \frac{\partial^{5} U_{1}}{\partial \theta \partial x^{4}}\right)
\end{aligned}
$$

$\omega^{2} \frac{\partial^{5} y}{\partial t \partial x^{4}}=\varepsilon A_{1} \Omega_{1}^{2} \cos \varphi Z_{1}-a \Omega_{1}^{3} \sin \varphi Z_{1}-\varepsilon B_{1} a \Omega_{1}^{2} \sin \varphi Z_{1}+\varepsilon \omega^{2} \frac{\partial}{\partial x^{4}} L_{1}\left[U_{1}\right]$

$$
\xi \omega^{2} \frac{\partial^{4} y}{\partial x^{4}}=\xi \omega^{2} a \cos \varphi \frac{\pi^{4}}{\ell^{4}} Z_{1}+\varepsilon \xi \omega^{2} \frac{\partial^{4} U_{1}}{\partial x^{4}}=\xi \Omega_{1}^{2} a \cos \varphi Z_{1}+\varepsilon \xi \omega^{2} \frac{\partial^{4} U_{1}}{\partial x^{4}} .
$$

Substituting expressions (11), (12), (13) and (14) into the equation (1) we obtain $\varepsilon \frac{\partial^{2} A_{1}}{\partial \psi^{2}}\left(\Omega_{1}-\frac{p}{q} \gamma\right)^{2} \cos \varphi Z_{1}-3 \varepsilon \frac{\partial A_{1}}{\partial \psi}\left(\Omega_{1}-\frac{p}{q} \gamma\right) \Omega_{1} \sin \varphi Z_{1}-3 \varepsilon A_{1} \Omega_{1}^{2} \cos \varphi Z_{1}$ $-\varepsilon \frac{\partial^{2} B_{1}}{\partial \psi^{2}}\left(\Omega_{1}-\frac{p}{q} \gamma\right)^{2} a \sin \varphi Z_{1}-3 \varepsilon \frac{\partial B_{1}}{\partial \psi}\left(\Omega_{1}-\frac{p}{q} \gamma\right) a \Omega_{1} \cos \varphi Z_{1}$

$+3 \varepsilon B_{1} a \Omega_{1}^{2} \sin \varphi Z_{1}+a \Omega_{1}^{3} \sin \varphi Z_{1}+\varepsilon L_{3}\left[U_{1}\right]+\varepsilon \xi \frac{\partial A_{1}}{\partial \psi}\left(\Omega_{1}-\frac{p}{q} \gamma\right) \cos \varphi Z_{1}$

$\left.-2 \varepsilon \xi A_{1} \Omega_{1} \sin \varphi Z_{1}-2 \varepsilon \xi B_{1} \Omega_{1} a \cos \varphi Z_{1}-\varepsilon \xi \frac{\partial B_{1}}{\partial \psi}\left(\Omega_{1}-\frac{p}{q} \gamma\right) a \sin \varphi Z_{1}\right]$

$-\xi a \Omega_{1}^{2} \cos \varphi Z_{1}+\varepsilon \xi L_{2}\left[U_{1}+\varepsilon A_{1} \Omega_{1}^{2} \cos \varphi Z_{1}-a \Omega_{1}^{3} \sin \varphi Z_{1}-\varepsilon B_{1} a \Omega_{1}^{2} \operatorname{sir} \varphi Z_{1}\right.$

$+\varepsilon \omega^{2} \frac{\partial}{\partial x^{4}} L_{1}\left[U_{1}\right]+\xi \Omega_{1}^{2} a \cos \varphi Z_{1}+\varepsilon \xi \omega^{2} \frac{\partial^{4} U_{:}}{\partial x^{4}}+\ldots=\varepsilon F\left(a \cos \varphi Z_{1},-a \Omega_{1} \sin \varphi, \ldots\right)$ 
Comparing the coefficients of like powers of $\varepsilon$ we have

$$
\begin{aligned}
& L_{3}\left[U_{1}\right]+\xi L_{2}\left[U_{1}\right]+\omega^{2} L_{1}\left[\frac{\partial^{4} U_{1}}{\partial x^{4}}\right]+\xi \omega^{2} \frac{U_{1}}{\partial x^{4}}+\left\{\left[\left(\Omega_{1}-\frac{p}{q} \gamma\right)^{2} \frac{\partial^{2} A_{1}}{\partial \psi^{2}}+\xi\left(\Omega_{1}-\frac{p}{q} \gamma\right)\right.\right. \\
& \left.\frac{\partial A_{1}}{\partial \psi}-3 a \Omega_{1}\left(\Omega_{1}-\frac{p}{q} \gamma\right) \frac{\partial B_{1}}{\partial \Psi}-2 \Omega_{1}^{2} A_{1}-2 \Omega_{1} \xi \alpha B_{1}\right] \cos \varphi+ \\
& {\left[-a\left(\Omega_{1}-\frac{p}{q} \gamma\right)^{2} \frac{\partial^{2} B_{1}}{\partial \psi^{2}}-\xi a\left(\Omega_{1}-\frac{p}{q} \gamma\right) \frac{\partial B_{1}}{\partial \Psi}-3 \Omega_{1}\left(\Omega_{1}-\frac{p}{q} \gamma\right) \frac{\partial A_{1}}{\partial \psi}\right]-2 \xi \Omega_{1} A_{1}} \\
& \left.\left.+2 a \Omega_{1}^{2} B_{1}\right] \sin \varphi U\right\} Z_{1}=F_{1}
\end{aligned}
$$

where the linear operators take the from

$$
\begin{aligned}
& L_{1}\left[U_{1}\right]=\left(\frac{\partial}{\partial \varphi} \Omega_{1}-\gamma \frac{\partial}{\partial \theta}\right) U_{1}, \\
& L_{2}\left[U_{1}\right]=\left(\Omega_{1} \frac{\partial}{\partial \varphi}+\gamma \frac{\partial}{\partial \theta}\right)^{2} U_{1}, \\
& L_{3}\left[U_{1}\right]=\left(\Omega_{1} \frac{\partial}{\partial \varphi}+\gamma \frac{\partial}{\partial \theta}\right)^{3} U_{1}
\end{aligned}
$$

The relevant homogeneous boundary conditions of the function $U_{1}$ are

$$
\left.U_{1}\right|_{x=0}=0,\left.\frac{\partial^{2} U_{1}}{\partial x^{2}}\right|_{x=0}=0,\left.\quad U_{1}\right|_{x=l}=0,\left.\frac{\partial^{2} U_{1}}{\partial x^{2}}\right|_{x=l}=0
$$

Now we expand the functions $U_{1}$ and $F_{1}$ into the series of functions $\left\{Z_{K}(x)\right\}$

$$
\begin{aligned}
& U_{1}=\sum_{k=1}^{\infty} U_{1 k}(a, \varphi, \theta) Z_{K}(x), \\
& F_{1}=\sum_{k=1}^{\infty} F_{1 k}(a, \varphi, \theta) Z_{K}(x), \\
& F_{1 k}=\int_{0}^{t} F_{1} Z_{k} d x / \int_{0}^{t} Z_{k}^{2}(x) d x .
\end{aligned}
$$

where $F_{1 K}(a, \varphi, \theta)$ are defined, still $U_{1 K}(a, \varphi, \theta)$ need to be determined.

We calculate some quantities in the equation (16)

$$
\begin{aligned}
& L_{3}\left[U_{1}\right]=\sum_{k=1}^{\infty} L_{3}\left[U_{1 k}\right] Z_{k}, \xi L_{2}\left[U_{1}\right]=\xi \sum_{k=1}^{\infty} L_{2}\left[U_{l k}\right] Z_{k} \\
& \omega^{2} L_{1}\left[\frac{\partial^{4} U_{1}}{\partial x^{4}}\right]=\sum_{k=1}^{\infty} L_{1}\left[U_{l k}\right] \Omega_{k}^{2} Z_{k}, \xi \omega^{2} \frac{\partial^{4} U_{1}}{\partial x^{4}}=\sum_{k=1}^{\infty} U_{l k} \xi \Omega_{k}^{2} Z_{k} .
\end{aligned}
$$


Substituting (15) into (16) we have

$$
\begin{aligned}
& \sum_{K=1}^{\infty}\left\{L_{3}\left[U_{1 K}\right]+\xi L_{2}\left[U_{1 K}\right]+\Omega_{K}^{2} L_{1}\left[U_{1 K}\right]+\xi \Omega_{K}^{2} U_{1 K}\right\} Z_{K}+ \\
& +\left[\left(\Omega_{1}-\frac{p}{q} \gamma\right)^{2} \frac{\partial^{2} A_{1}}{\partial \psi^{2}}+\xi\left(\Omega_{1}-\frac{p}{q} \gamma\right) \frac{\partial A_{1}}{\partial \psi}-2 a \Omega_{1}\left(\Omega_{1}-\frac{p}{q} \gamma\right) \frac{\partial B_{1}}{\partial \psi}\right. \\
& \left.-2 \Omega_{1}^{2} A_{1}-2 \xi \Omega_{1} a B_{1}\right] \cos \varphi+\left[-a\left(\Omega_{1}-\frac{p}{q} \gamma\right)^{2} \frac{\partial^{2} B_{1}}{\partial \psi^{2}}-\xi a\left(\Omega_{1}-\frac{p}{q} \gamma\right) \frac{\partial B_{1}}{\partial \psi}\right. \\
& \left.\left.-3 \Omega_{1}\left(\Omega_{1}-\frac{p}{q} \gamma\right) \frac{\partial A_{1}}{\partial \psi}-2 \xi \Omega_{1} A_{1}+2 a \Omega_{1}^{2} B_{1}\right] \sin \varphi\right\} Z_{1}=\sum_{K=1}^{\infty} F_{1 K} Z_{K}
\end{aligned}
$$

In the case of $k=1$ we obtain the following equations

$$
\begin{aligned}
& L_{3}\left[U_{11}\right]+\xi L_{2}\left[U_{11}\right]+\Omega_{1}^{2} L_{2}\left[U_{11}\right]+\xi \Omega_{1}^{2} U_{11} \\
& =\left[\left(\Omega_{1}-\frac{p}{\gamma}\right)^{2} \frac{\partial^{2} A_{1}}{\partial \psi^{2}}+\xi\left(\Omega_{1}-\frac{p}{q} \gamma\right) \frac{\partial A_{1}}{\partial \psi}-3 a \Omega_{1}\left(\Omega_{1}-\frac{p}{q} \gamma\right) \frac{\partial B_{1}}{\partial \psi}\right. \\
& \left.-2 \Omega_{1}^{2} A_{1}-2 \xi a \Omega_{1} B_{1}\right] \cos \varphi+\left[a\left(\Omega_{1}-\frac{p}{q} \gamma\right)^{2} \frac{\partial B_{1}}{\partial \psi^{2}}+\xi a\left(\Omega_{1}-\frac{p}{q} \gamma\right) \frac{\partial B_{1}}{\partial \psi}\right. \\
& \left.+3 \Omega_{1}\left(\Omega_{1}-\frac{p}{q} \lambda\right) \frac{\partial A_{1}}{\partial \psi}+2 \xi \Omega_{1} A_{1}-2 a \Omega_{1}^{2} B_{1}\right] \sin \varphi+F_{11}, \\
& L_{3}\left[U_{1 k}\right]+\xi L_{2}\left[U_{1 k}\right]+\Omega_{1}^{2} L_{2}\left[U_{1 k}\right]+\xi \Omega_{1}^{2} U_{1 k}=F_{1 k} \quad(k=2,3 \ldots)
\end{aligned}
$$

In order to find the functions $U_{1 k}(a, \varphi, \theta)$ we again expand $U_{1 k}$ and $U_{1 k}(a, \varphi, \theta)$ into the Fourier series

$$
U_{1 k}(a, \varphi, \theta)=\sum_{m, n=-\infty}^{+\infty} U_{n m}^{(k)}(a) e^{i(n \theta+m \varphi)},
$$

where $U_{n m}^{(k)}(a)$ need to be determined

$$
\begin{aligned}
& F_{1 k}(a, \varphi, \theta)=\sum_{m, n=-\infty}^{+\infty} F_{n m}^{(k)}(a) e^{i(n \theta+m \varphi)}, \\
& F_{m n}^{(k)}(a)=\frac{1}{4 \pi^{2}} \int_{0}^{2 \pi} \int_{0}^{2 \pi} F_{1 k} e^{-i(n \theta+m \varphi)} d \theta d \varphi .
\end{aligned}
$$

From (24) and noting (17) we get

$$
\begin{aligned}
& L_{1}\left[U_{1 k}\right]=L_{1}\left[\sum_{m, n} U_{m n}^{(k)}(a) e^{i(m \varphi+n \theta)}\right] \sum_{m n} U_{m n}^{(k)}(a) L_{1}\left[e^{i(m \varphi+n \theta)}\right], \\
& L_{1}\left[U_{1 k}\right]=\sum_{m n} i\left(m \Omega_{1}+n \gamma\right) U_{m n}^{(k)}(a) e^{i(m \varphi+n \theta)} .
\end{aligned}
$$




$$
\begin{aligned}
& L_{2}\left[U_{1 k}\right]=L_{2}\left[\sum_{m, n} U_{m n}^{(k)}(a) e^{i(m \varphi+n \theta)}\right] \sum_{m n} U_{m n}^{(k)}(a) L_{2}\left[e^{i(m \varphi+n \theta)}\right], \\
& L_{2}\left[U_{1 k}\right]=\sum_{m n} i^{2}\left(m \Omega_{1}+n \gamma\right)^{2} U_{m n}^{(k)}(a) e^{i(m \varphi+n \theta)} . \\
& L_{3}\left[U_{l k}\right]=L_{3}\left[\sum_{m, n} U_{m n}^{(k)}(a) e^{i(m \varphi+n \theta)}\right]=\sum_{m n} U_{m n}^{(k)}(a) L_{3}\left[e^{i(m \phi+n \theta)}\right], \\
& L_{3}\left[U_{l k}\right]=\sum_{m n} i^{3}\left(m \Omega_{1}+n \gamma\right)^{3} U_{m n}^{(k)}(a) e^{i(m \varphi+n \gamma)}
\end{aligned}
$$

Substituting (25) - (28) into (23) we obtain

$$
\begin{aligned}
& \sum_{m n} i^{3}\left(m \Omega_{1}+n \gamma \gamma^{3} U_{m n}^{(k)}(a) e^{i(m \varphi+n \theta \theta}+\sum_{m n} \xi i i^{2}\left(m \Omega_{1}+\gamma \gamma^{2} U_{m n}^{(k)}(a) e^{i(m \varphi+n \theta \theta)}\right.\right. \\
& +\sum_{m n} \Omega_{k}^{2} i\left(m \Omega_{1}+n \theta \theta\right)_{m n}^{(k)}(a) e^{i(m \varphi+n \theta \theta}+\xi \Omega_{k}^{2} \sum_{m n} U_{m n}^{(k)}(a) e^{i(m \varphi+n \theta \theta} \\
& =\sum_{m n} F_{m n}^{(k)}(a) e^{i(m \varphi+n \theta \theta} .
\end{aligned}
$$

From the above equation, after calculating we get the following expresstion

$$
\begin{aligned}
& {\left[i^{3}\left(m \Omega_{1}^{+} n \gamma\right)^{3}+\xi i^{2}\left(m \Omega_{1}+n \gamma\right)^{2}+i \Omega_{k}^{2}\left(m \Omega_{1}+n \gamma\right)+\xi \Omega_{k}^{2}\right] U_{m n}^{(k)}(a)=F_{m n}^{(k)}(a),} \\
& \left\{i ^ { 2 } ( m \Omega _ { 1 } + n \gamma ) ^ { 2 } \left[\left(\xi+i\left(m \Omega_{1}+n \gamma\right)\right]+\Omega_{k}^{2}\left[\xi+i\left(m \Omega_{1}+n \gamma\right)\right] U_{m n}^{k}(a)=F_{m n}^{(k)}(a),\right.\right. \\
& \left\{\begin{array}{l}
{\left[\Omega_{k}^{2}-\left(m \Omega_{1}+n \gamma\right]\left[\xi+i\left(m \Omega_{1}^{+} n \gamma\right] U_{m n}^{(k)}(a)=F_{m n}^{(k)}(a),\right.\right.} \\
U_{m n}^{(k)}(a)=\begin{array}{l}
F_{m n}^{(k)}(a) \\
{\left[\xi+i\left(m \Omega_{1}+n \gamma\right)\right]\left[\Omega_{k}^{2}-\left(m \Omega_{1}+n \gamma\right)^{2}\right]}
\end{array}
\end{array}\right.
\end{aligned}
$$

Substituting (29) into the expression (24) the funtions $U_{1 k}(a, \varphi, \theta)$ are determined

$$
U_{l k}(a, \varphi, \theta)=\sum_{m n} \frac{\int_{0}^{2 \pi} \int_{0}^{2 \pi} F_{l k}(a, \varphi, \theta) e^{-i(m \varphi+n \theta)} d \varphi d \theta}{4 \pi^{2}\left[\xi+i\left(m \Omega_{1}+n \gamma\right)\right]\left[\Omega_{k}^{2}-\left(m \Omega_{1}+n \gamma\right)^{2}\right]}
$$

It is seen that when $k=1$ we have

$$
\begin{aligned}
& {\left[\Omega_{1}^{4}-\left(m \Omega_{1}+n \gamma^{2}\right] \neq 0\right.} \\
& {\left[\Omega_{1}(m \pm 1)+n \gamma\right] \neq 0,}
\end{aligned}
$$

Substituting $\Omega_{1} \approx \frac{p}{q} \gamma$ into the above inequalities yields

$$
[p(m \pm 1)+n q] \neq 0 .
$$


Substituting (30) into (29) we obtain the expression for the function $U_{1}=U_{1}(x, a, \varphi, \theta)$ as follows

$$
U_{1}=\sum_{m n} \sum_{k=1}^{\infty}\left\{\frac{\int_{0}^{2 \pi} \int_{0}^{2 \pi}\left[\int_{0}^{l} F_{1} Z_{k}(x) d x\right] e^{-i(m \varphi+n \theta)} d \varphi d \theta \cdot Z_{k}(x) e^{i(m \varphi+n \theta)}}{4 \pi^{2}\left[\xi+i\left(m \Omega_{1+n \gamma)}\right]\left[\Omega_{k}^{2}-\left(m \Omega_{1}+n \gamma\right)^{2}\right] \int_{0}^{l} Z_{k}^{2}(x) d x\right.}\right\}
$$

When $k=1$ we have

$$
[p(m \pm 1)+n p] \neq 0
$$

In order to determine $A_{1}, B_{1}$, now we substitute (26), (27) and (28) into (23), supposing that $U_{1}$ does not contain $\cos \varphi, \sin \varphi$. It means

$$
\left\langle U_{1} \cos \varphi\right\rangle=0,\left\langle U_{1} \sin \varphi\right\rangle=0
$$

Therefore the equation (22) becomes

$$
\begin{aligned}
& -\left[\left(\Omega_{1}-\frac{p}{q} \gamma\right)^{2} \frac{\partial^{2} A_{1}}{\partial \psi^{2}}+\xi\left(\Omega_{1}-\frac{p}{q} \gamma\right) \frac{\partial A_{1}}{\partial \Psi}-3 a \Omega_{1}\left(\Omega_{1}-\frac{p}{q} \gamma\right) \frac{\partial B_{1}}{\partial \psi}\right. \\
& \left.-2 \Omega_{1}^{2} A-2 \xi \Omega_{1} a B_{1}\right] \cos \varphi\left[a\left(\Omega_{1}-\frac{p}{q} \gamma\right)^{2} \frac{\partial^{2} B_{1}}{\partial \psi^{2}}+\xi a\left(\Omega_{1}-\frac{p}{q} \gamma\right) \frac{\partial B_{1}}{\partial \psi}\right. \\
& \left.-3 \Omega_{1}\left(\Omega_{1}-\frac{p}{q} \gamma\right) \frac{\partial A_{1}}{\partial \psi}+2 \xi \Omega_{1} A_{a}-2 a \Omega_{1}^{2} B_{1}\right] \sin \varphi+F_{11}=0
\end{aligned}
$$

where

$$
\begin{aligned}
& F_{11}(a, \varphi, \theta)=\sum_{m n} F_{m n}^{(1)}(a) e^{1(m \varphi+n \theta \theta}, \\
& F_{m n}^{(1)}(a)=\frac{1}{4 \pi^{2}} \int_{0}^{2 \pi} \int_{0}^{2 \pi} F_{11} e^{-i(m \varphi+n \theta \theta} d \varphi d \theta,
\end{aligned}
$$

In the expresstions (34), the terms contain $\cos \varphi, \sin \varphi$ corresponding to the values $m, n=-\infty \div+\infty$, for which

$$
m \varphi+n \theta= \pm \varphi+\mu
$$

where the quantity $\mu$ needs be determined. Noting (7) we have

$$
(m \mp 1) \varphi+n \theta=\mu \rightarrow(m \mp 1) \frac{p}{q} \theta+(m \mp 1) \psi+n \theta=\mu,
$$

Putting:

We have

$$
\frac{(m \pm 1)}{q}=r \rightarrow m=r q \pm 1
$$

$$
\begin{gathered}
r p \theta+r q \psi+n \theta=\mu \rightarrow r p \theta+n \theta=0 \rightarrow n=-r p \\
\mu=r q \psi(r=-\infty \div+\infty)
\end{gathered}
$$

where $r$ is a proportional coefficient. 
Now we have to calculate the quantity in (34) in two cases where

$$
\begin{aligned}
& m \varphi+n \theta=+\varphi+\mu \\
& m \varphi+n \theta=-\varphi+\mu
\end{aligned}
$$

Substituting (39) into (34) and noting (38) we obtain

$$
\begin{aligned}
F_{11}(a, \varphi, \theta)= & \sum_{r} e^{-i r q \psi} e^{i \varphi} \frac{1}{4 \pi^{2}} \int_{0}^{2 \pi} \int_{0}^{2 \pi} F_{11} e^{-i r q \psi} e^{-i \varphi} d \varphi d \theta \\
& +\sum_{r} e^{i r q \psi} e^{-i r} \frac{1}{4 \pi^{2}} \int_{0}^{2 \pi} \int_{0}^{2 \pi} F_{11} e^{-i r q \psi} e^{+i \varphi} d \varphi d \theta \\
F_{11}= & \frac{1}{4 \pi^{2}} \sum_{r} e^{i r q \psi}\left\{(\cos \varphi+i \sin \varphi) \int_{0}^{2 \pi} \int_{0}^{2 \pi} F_{11} e^{-i r q \psi}(\cos \varphi-i \sin \varphi) d \varphi d \theta\right\} \\
& \left.+(\cos \varphi-i \sin \varphi) \int_{0}^{2 \pi} \int_{0}^{2 \pi} F_{11} e^{-i r q \psi}(\cos \varphi-i \sin \varphi) d \varphi d \theta\right\}
\end{aligned}
$$

The expression (40) can be written in the form

$$
\begin{aligned}
F_{11}= & \frac{1}{4 \pi^{2}} \sum_{r} e^{i r q \psi}\left\{\int_{0}^{2 \pi} \int_{0}^{2 \pi} F_{11} e^{-i r q \psi}[(\cos \varphi d \varphi d \theta) \cos \varphi-i(\sin \varphi d \varphi d \theta) \cos \varphi\right. \\
& +(\sin \varphi d \varphi d \theta) \sin \varphi+i(\cos \varphi d \varphi d \theta) \sin \varphi+(\cos \varphi d \varphi d \theta) \sin \varphi \\
& +i(\sin \varphi d \varphi d \theta) \cos \varphi-i(\cos \varphi d \varphi d \theta) \sin \varphi+(\sin \varphi d \varphi d \theta) \sin \varphi]\}
\end{aligned}
$$

It is seen that the function $F_{11}$ is rewritten

$$
\begin{aligned}
F_{11}= & \frac{2}{4 \pi^{2}} \sum_{r} e^{i r q \psi}\left\{\int_{0}^{2 \pi} \int_{0}^{2 \pi} F_{11} e^{-i r q \psi} \cos \varphi d \varphi d \theta \cos \varphi+\int_{0}^{2 \pi} \int_{0}^{2 \pi} F_{11} e^{i r q \psi}+\sin \varphi d \varphi d \theta \sin \varphi\right\} \\
= & {\left[\frac{1}{2 \pi^{2}} \sum_{r} e^{i r q \psi} \int_{0}^{2 \pi} \int_{0}^{2 \pi} F_{11} e^{-i r q \psi} \cos \varphi d \varphi d \theta\right] \cos \varphi } \\
& +\left[\frac{1}{2 \pi^{2}} \sum_{r} e^{i r q \psi} \int_{0}^{2 \pi} \int_{0}^{2 \pi} F_{11} e^{-i r q \psi} \sin \varphi d \varphi d \theta\right] \sin \varphi
\end{aligned}
$$


Substituting (41) into (33) and then comparing the coefficients of $\cos \varphi, \sin \varphi$ we get

$$
\begin{aligned}
& \left(\Omega_{1}-\frac{p}{q} \gamma\right)^{2} \frac{\partial^{2} A_{1}}{\partial \psi^{2}}+\xi\left(\Omega_{1}-\frac{p}{q} \gamma\right) \frac{\partial^{2} A_{1}}{\partial \psi}-3 a \Omega_{1}\left(\Omega_{1}-\frac{p}{q} \gamma\right) \frac{\partial B_{1}}{\partial \psi}-2 \Omega^{2} A_{1}-2 \xi \Omega_{1} a B_{1} \\
& =\frac{1}{2 \pi^{2}} \sum_{\gamma} e^{i r q \psi} \int_{0}^{2 \pi} \int_{0}^{2 \pi} F_{11} e^{-i r q \psi} \cos \varphi d \varphi d \theta\left[a\left(\Omega_{1}-\frac{p}{q} \gamma\right)^{2} \frac{\partial^{2} B_{1}}{\partial \psi^{2}}+\xi a\left(\Omega_{1}-\frac{p}{q} \gamma\right) \frac{\partial B_{1}}{\partial \psi}\right. \\
& \left.+3 \Omega_{1}\left(\Omega_{1}-\frac{p}{q} \gamma\right) \frac{\partial A_{1}}{\partial \psi}+2 \xi \Omega_{1} A_{1}-2 a \Omega_{1}^{2} B_{1}\right]=-\frac{1}{2 \pi^{2}} \sum_{r} e^{i r q \psi} \int_{0}^{2 \pi} \int_{0}^{2 \pi} F_{11} e^{-i r q \psi} \sin \varphi d \varphi d \theta
\end{aligned}
$$

It is supposed that there exists a resonance relation

$$
\Omega_{1} \approx \frac{p}{q} \gamma+\varepsilon \Delta
$$

Substituting (43) into (42), neglecting the small terms of $\varepsilon^{2}$ order we get

$$
\begin{aligned}
\Omega_{1}^{2} A_{1}+\xi \Omega_{1} a B_{1} & =-\frac{1}{4 \pi^{2}} \sum_{r} e^{e i r q \psi} \int_{0}^{2 \pi} \int_{0}^{2 \pi} F_{11} e^{-i r q\left(\varphi-\frac{p}{q} \theta\right)} \cos \varphi d \varphi d \theta=G \\
\xi \Omega_{1} A_{1}-\Omega_{11}^{2} a B_{1} & =-\frac{1}{4 \pi^{2}} \sum_{r} e^{e i r q \psi} \int_{0}^{2 \pi} \int_{0}^{2 \pi} F_{11} e^{-i r q\left(\varphi-\frac{p}{q} \theta\right)} \sin \varphi d \varphi d \theta=H
\end{aligned}
$$

It is seen from (??) that $A_{1}$ and $B_{1}$ are determined

$$
A_{1}=\frac{\left(\Omega_{1} G+\xi H\right)}{\Omega_{1}\left(\Omega_{1}^{2}+\xi^{2}\right.} ; \quad B_{1}=\frac{\xi G-\Omega_{1} H}{a \Omega_{1}\left(\Omega_{1}^{2}+\xi^{2}\right)}
$$

Thus, in the improved first approximation the partial solution of equation (6) is determined

$$
\begin{aligned}
& y=a \cos \left(\frac{p}{q} \theta+\psi\right) \sin \frac{\pi x}{\ell} \\
& +\varepsilon \sum_{m n} \sum_{k=1}^{\infty}\left\{\frac{\int_{0}^{2 \pi} \int_{0}^{2 \pi}\left[\int_{0}^{2 \pi} F_{1}(x, a, \varphi, \theta) \sin \frac{k \pi \pi}{\ell} d x\right] e^{-i(m \varphi+n \theta \theta} d \varphi d \theta \theta \sin \frac{k \pi \pi}{\ell} e^{i(m \varphi+n \theta \theta}}{4 \pi^{2}\left[\xi+i\left(m \Omega_{1}+n \gamma \gamma\right]\left[\Omega_{k}^{2}-\left(m \Omega_{1}+n \gamma\right)^{2}\right] \int_{0}^{\ell} \sin ^{2} \frac{k \pi \pi}{\ell} d x\right.}\right\}
\end{aligned}
$$

When $k=1$ we have $\{\mathrm{p}(\mathrm{m} \pm 1)+n q] \neq 0$

$a$ and $\psi$ are determined from the following equations

$$
\begin{aligned}
& \frac{d a}{d t}=\varepsilon A_{1}(a, \psi)+\varepsilon^{2} \ldots \\
& \frac{d \psi}{d t}=\left(\Omega_{1}-\frac{p}{q} \gamma\right)+\varepsilon B_{1}(a, \psi)+\varepsilon^{2} \ldots
\end{aligned}
$$


However, practically one only needs to find the particular solution in the first approximation

$$
y(x, t)=a \cos \left(\frac{p}{q} \theta+\eta\right) \sin \frac{\pi x}{l}
$$

\section{CONCLUSION}

In this work, the authors have investigated the nonlinear oscillation of creepy elastic beam in the ron-autonmous case with the homogeneous linear boundary condition. It is easy seen that the motion of the creepy elastic beam is described by the partial differential equation of the third order with respect to argument.

The solution of this equation has been constructed by the asymptctic method for the high order systems. In the improved approximation the solution of the boundary value problem has been determined.

\section{ACKNOWLEDGEMENT}

Support frm the council for natural sciences of Vietnam is gratefully acknowledge.

\section{REFERENCES}

[1] H. V. Da. Vibration of thin rectangular viscous elastic plate, In Ucrainian Journal of Applied Mechanics 19 (12) 120-124 (in Russian).

[2] H.V. Da., Parametric vibration of beam with the creep of material and nonlinear boudary conditions. In Romonia Journal of Applied Mechanics 29 (5), Bucarest (1984) 457-552 (in Russian).

[3] H.V. Da; Tr. D. Son, Vibration of creep beam, In Science Technology Journal 5 (47), (2007) 117-131.

[4] N.V. Dao., Fundamental methods of the theory of nonlinear oscillations. Higher and secondary education Publication, Hanoi, 1971 (in Victiamese).

[5] N.V. Dao, Nonlinear oscillations of high order systems, National center fcr scientific research of Vietnam, Hanoi, 1979.

[6] Mitropolskii Yu. A, Asymptotic solutions of equations with partial derivatives, Kiev, Ucraina, 1968 (in Russian).

[7] Rgianisun. A.P., Theory of creep. Civil engineering Publication, Moscow, 1968 (in Russian).

[8] Timoshenko. Vibration problems in Engineering. Science Publication. Hanoi, 1963 (in Vietnamese).

Received June 21, 2009

\section{NGHIỆM TIỆM CẬN CỦA PHƯƠNG TRÌNH ĐẠO HÀM RIÊNG CẤP CAO}

Trong bài báo này, các tác giả xây dựng mô hình nghiệm tiệm cận của phương trình đạo hàm riêng cấp cao mô tả dao động của dầm đàn hồi phi tuyến từ biến trong trường hợp hệ không ôtônôm. Trong xấp xỉ thứ nhất hoàn thiện nghiệm tiệm cận của bài toán biên đó được xác định. 